\title{
NECESSARY OPTIMALITY CONDITIONS FOR A PROBLEM WITH COSTS OF RAPID VARIATION OF CONTROL
}

\author{
K. KIBALCZYC AND S. WALCZAK'
}

(Received 23 April 1982; revised 16 March 1983)

\begin{abstract}
In this paper a control problem with a cost functional depending on the number of switchings and on the speed of alterations of control is considered. Necessary conditions for the existence of an optimal solution are given.
\end{abstract}

\section{Introduction}

Denote by $U$ a set of piecewise continuous vector functions $u(\cdot)=$ $\left(u^{1}(\cdot), \ldots, u^{r}(\cdot)\right)$ defined on the interval $[0, T]$, whose values at the instant $t$ belong to a non-empty compact set $M$ contained in $\mathbf{R}^{r}$. Besides, let us assume that, for the functions $u(\cdot)$ belonging to $U$, the condition: $S(u)<\infty$ is satisfied, where $S(u)$ is defined by the formula

$$
S(u)=\sup \left\{\frac{\left|u(t)-u\left(t^{\prime}\right)\right|}{\left|t-t^{\prime}\right|} ; t \neq t^{\prime}, u \text { continuous on }\left[t, t^{\prime}\right]\right\} \text {. }
$$

The set $U$ will be called a set of admissible controls and its elements admissible controls.

Consider the following

Problem I. Determine the minimal value of the functional

$$
I(x, u)=\int_{0}^{T} f_{0}(t, x(t), u(t)) d t+\Phi(u)
$$

\footnotetext{
Institute of Mathematics, University of Lodź, Poland.

( Copyright Australian Mathematical Society 1984, Serial-fee code 0334-2700/84
} 
under the conditions

$$
\begin{aligned}
& \dot{x}(t)=f(t, x(t), u(t)), \\
& x(0)=x_{0}, \\
& u(\cdot) \in U,
\end{aligned}
$$

where $f_{0}:[0, T] \times \mathbf{R}^{n} \times \mathbf{R}^{r} \rightarrow \mathbf{R}, f:[0, T] \times \mathbf{R}^{n} \times \mathbf{R}^{r} \rightarrow \mathbf{R}^{n}, x(\cdot) \in W_{1,1}^{n}([0, T])$, $x_{0} \in \mathbf{R}^{n}, W_{1,1}^{n}([0, T])$ stands for the space of absolutely continuous functions with norm

$$
\|x\|=|x(0)|+\int_{0}^{T}|\dot{x}(t)| d t .
$$

The functional $\Phi(u)$ is defined by the formula

$$
\Phi(u)=\gamma_{1} N(u)+\gamma_{2} S(u)
$$

where $\gamma_{1}>0, \gamma_{2}>0, N(u)$ denotes the number of points of discontinuity of the function $u(\cdot)$ on $[0, T]$, and $S(u)$ is defined by (1).

A problem like this was formulated by E. S. Noussair [3]. In the paper just mentioned the author proved a theorem on the existence of a solution to Problem $I$ in the class of piecewise continuous functions. The present paper includes a necessary condition for optimality which has been obtained on the basis of the Ioffe-Tikhomirov extremum principle ([2], Section 1.1).

\section{A necessary condition for optimality}

Let $\left(x_{*}, u_{*}\right)$ be a solution to Problem I. Such a solution exists if the assumptions of Theorem 3 are satisfied ( $c f$. [3]). Assume what follows:

$1^{\circ}$ there exists a summable function $\alpha(\cdot)$ such that $f_{0}(t, x(t), u(t)) \geqslant \alpha(t)$ for any pair $(x, u)$ satisfying (3)-(5) and for almost all $t \in[0, T]$,

$2^{\circ}$ the functions $f_{0}(t, x, u), f(t, x, u)$ are continuous with respect to the group of variables $(t, x, u)$, continuously differentiable with respect to $x$ and differentiable with respect to $u$,

$3^{\circ} f_{0}$ is convex with respect to $u$ with any $x$ and $t \in[0, T]$,

$4^{\circ}$ there exists a neighbourhood $V \subset W_{1,1}^{n}([0, T])$ of the point $x_{*}$ such that for any $x \in V$, any $u_{1}, u_{2} \in U_{p}$ and any $\alpha \in[0,1]$ there exists some $u \in U_{p}$ such that

$$
f(t, x(t), u(t))=\alpha f\left(t, x(t), u_{1}(t)\right)+(1-\alpha) f\left(t, x(t), u_{2}(t)\right)
$$

for each $t \in[0, T]$, where $U_{p} \subset U$ is a set of functions that have $p$ fixed points of discontinuity,

$5^{\circ}$ the set $M$, occurring in the definition of the set of admissible controls $U$, is a 
perpendicular parallelepiped contained in $\mathbf{R}^{r}$, defined as follows:

$$
M=\left\{z=\left(z^{1}, \ldots, z^{r}\right) \in \mathbf{R}^{r} ; z^{i} \in\left[\alpha^{i}, \beta^{i}\right], i=1, \ldots, r\right\},
$$

where $-\infty<\alpha_{i}<\beta_{i}<\infty$ for $i=1,2, \ldots, r$.

Remark 1. Conditions $1^{\circ}, 2^{\circ}, 3^{\circ}$ and $5^{\circ}$ are typical assumptions which occur in many theorems of optimization theory. Condition $4^{\circ}$ has been introduced here in order that the Ioffe-Tikhomirov extremum principle could be applied. It is easily noticed that this assumption is satisfied by all systems linear with respect to the control, i.e. systems of the form

$$
f(t, x, u)=g_{1}(t, x) u+g_{2}(t, x),
$$

where $u \in U, u(t) \in M$, and $M$ is a convex set.

Denote by $H$ a Hamilton function

$$
H\left(\lambda_{0}, \psi, x, u, t\right)=\left(\psi(t), f(t, x(t), u(t))-\lambda_{0} f_{0}(t, x(t), u(t)),\right.
$$

where $\psi(t)$ is an absolutely continuous function on $[0, T]$ satisfying the conjugate equation

$$
\frac{d \psi(t)}{d t}=-f_{x}^{*}(t, x(t), u(t)) \psi(t)+\lambda_{0} f_{0_{x}}(t, x(t), u(t))
$$

and the condition $\psi(T)=0$, while $\lambda_{0}$ is some non-negative constant.

In the first place, let us consider the case when $u$ is a scalar function, that is, $r=1$.

We shall prove the following

THEOREM 1. If $u_{*}(\cdot)$ is an optimal control in Problem I and

1. conditions $\left(1^{\circ}-5^{\circ}\right)$ are satisfied,

2. $H_{u}\left(\lambda_{0}, \psi(t), x_{*}(t), u_{*}(t), t\right) \neq 0$ for almost all $t \in[0, T]$, then the optimal control $u_{*}(\cdot)$ is a piecewise constant function assuming values equal to $\alpha^{1}$ or $\beta^{1}$ only, and the number of points of discontinuity $N\left(u_{*}\right)$ can be estimated as follows

$$
N\left(u_{*}\right) \leqslant N\left(u_{0}\right)+\frac{c-\alpha_{0}}{\gamma_{1}},
$$

where $u_{0}(\cdot)$ is some admissible control,

$$
c=\int_{0}^{T} f_{0}\left(t, x_{0}(t), u_{0}(t)\right) d t+\gamma_{2} S\left(u_{0}\right), \quad \alpha_{0}=\int_{0}^{T} \alpha(t) d t .
$$

Proof. Denote $X=W_{1,1}^{n}([0, T]), Y=L_{1}^{n}([0, T]) \times \mathbf{R}^{n}$. Let $F: X \times U \rightarrow Y$ be an operator defined by the equality

$$
[F(x(\cdot), u(\cdot))](t)=\left(\dot{x}(t)-f(t, x(t), u(t)), x(0)-x_{0}\right) .
$$


Then Problem I can be formulated in the equivalent form of:

Problem II. Determine the minimal value of the functional

$$
I(x, u)=\int_{0}^{T} f_{0}(t, x(t), u(t)) d t+\Phi(u),
$$

in the set $X \times U$ under the conditions

$$
F(x, u)=0, \quad u(\cdot) \in U .
$$

Let $u_{*}(\cdot)$ be an optimal control in Problem I (Problem II). Denote by $t_{1}, t_{2}, \ldots, t_{z}$ points of discontinuity of the function $u_{*}(\cdot)$ on $[0, T]$. Note that $Z$ is a finite number. Indeed, from (2) we have

$$
\begin{aligned}
I\left(x_{*}, u_{*}\right) & =\int_{0}^{T} f_{0}\left(t, x_{*}, u_{*}\right) d t+\gamma_{1} N\left(u_{*}\right)+\gamma_{2} S\left(u_{*}\right) \\
& \leqslant I(0,0)=\int_{0}^{T} f_{0}(t, 0,0) d t .
\end{aligned}
$$

Making use of assumption $1^{\circ}$, we obtain the following estimate

$$
0 \leqslant Z=N\left(u_{*}\right) \leqslant \frac{1}{\gamma_{1}}\left[a+\alpha_{0}-\gamma_{2} S\left(u_{*}\right)\right] \leqslant \frac{1}{\gamma_{1}}\left[a+\alpha_{0}\right]<\infty,
$$

where

$$
a=\int_{0}^{T} f_{0}(t, 0,0) d t, \quad \alpha_{0}=\int_{0}^{T} \alpha(t) d t .
$$

Let $U_{z}$ stand for a subset of $U$, composed of functions possessing exactly $Z$ fixed points of discontinuity $t_{1}, t_{2}, \ldots, t_{z}$.

It is easily seen that, if $u_{*}(\cdot)$ is an optimal control in Problem II, it is also optimal for the following Problem III (but not inversely, in general).

Problem III. Find the minimal value of the functional

$$
I(x, u)=\int_{0}^{T} f_{0}(t, x(t), u(t)) d t+\gamma_{1} N(u)+\gamma_{2} S(u)
$$

under the conditions

$$
F(x, u)=0, \quad u(\cdot) \in U_{z} .
$$

For $u(\cdot)$ belonging to the set $U_{z}$, the $N(u)$ is constant and equal to $Z$; consequently, $u_{*}(\cdot)$ is also a solution to

Problem IV. Find the minimum of the functional

$$
\bar{I}(x, u)=\int_{0}^{T} f_{0}(t, x(t), u(t)) d t+\gamma_{2} S(u)
$$

under the conditions

$$
F(x, u)=0, \quad u(\cdot) \in U_{z} .
$$


And thus, if $u_{*}(\cdot)$ is an optimal control in Problem I, it is such for Problem IV.

To Problem IV we shall now apply the extremum principle ([2], Chapter 1).

The functional $S(u)$ is convex on $U_{z}$. Indeed, let $t, t^{\prime}$ be any points belonging to the interval of continuity of the functions $u_{1}(\cdot)$ and $u_{2}(\cdot)$. We have

$$
\begin{aligned}
S\left(\lambda u_{1}+(1-\lambda) u_{2}\right)= & \sup \left\{\mid \lambda u_{1}(t)+(1-\lambda) u_{2}(t)\right. \\
& \left.-\lambda u_{1}\left(t^{\prime}\right)-(1-\lambda) u_{2}\left(t^{\prime}\right)|/| t-t^{\prime} \mid\right\} \\
\leqslant & \lambda \sup \left\{\left|u_{1}(t)-u_{1}\left(t^{\prime}\right)\right| /\left|t-t^{\prime}\right|\right\} \\
& +(1-\lambda) \sup \left\{\left|u_{2}(t)-u_{2}\left(t^{\prime}\right)\right| /\left|t-t^{\prime}\right|\right\} \\
= & \lambda S\left(u_{1}\right)+(1-\lambda) S\left(u_{2}\right),
\end{aligned}
$$

where $\lambda \in[0,1]$. Consequently, the functional $\bar{I}(x, u)$ is convex with respect to $u$ with any $x \in V$.

The mapping $F$ is decomposed into mappings $F_{1}: X \times U \rightarrow Y_{1}=L_{1}^{n}[0, T]$ and $h_{1}: X \times U \rightarrow \mathbf{R}^{n}$, where

$$
\left[F_{1}(x(\cdot), u(\cdot))\right](t)=\dot{x}(t)-f(t, x(t), u(t)), \quad h_{1}(x(\cdot), u(\cdot))=x(0)-x_{0} .
$$

The mapping $F_{1}$ possesses a continuous Fréchet derivative with respect to $x$ for any $u(\cdot) \in U$, equal at the point $\left(x_{*}, u_{*}\right)$ to

$$
\left[F_{1_{x}}\left(x_{*}(\cdot), u_{*}(\cdot)\right) \bar{x}(\cdot)\right](t)=\dot{\bar{x}}(t)-f_{x}\left(t, x_{*}(t), u_{*}(t)\right) \bar{x}(t),
$$

where $\bar{x}(\cdot) \in W_{1,1}^{n}([0, T])$. The mapping $F_{1}$ is regular at the point $x_{*}$ because the equation

$$
\dot{\bar{x}}(t)-f_{x}\left(t, x_{*}(t), u_{*}(t)\right) \bar{x}(t)=y(t)
$$

possesses the solution $\bar{x}(t) \in W_{1,1}^{n}([0, T])$ for each

$$
y(\cdot) \in L_{1}^{n}([0, T]), \quad([2], \text { Section 0.2). }
$$

The functional $\bar{I}(x, u)=\int_{0}^{T} f_{0}(t, x(t), u(t)) d t+\gamma_{2} S(u)$ is also continuously Fréchet differentiable with respect to $x$ with each fixed $u \in U$, and its derivative at the point $\left(x_{*}, u_{*}\right)$ is expressed by the formula

$$
\bar{I}_{x}\left(x_{*}(\cdot), u_{*}(\cdot)\right) \bar{x}(\cdot)(t)=\int_{0}^{T}\left(f_{0_{x}}\left(t, x_{*}(t), u_{*}(t)\right), \bar{x}(t)\right) d t .
$$

In view of the above, all assumptions of the extremum principle are satisfied.

The Lagrange function for Problem IV has the form

$$
\mathcal{L}\left(x, u, \lambda_{0}, \lambda_{1}, y^{*}\right)=\lambda_{0} \bar{I}(x, u)+\left(\lambda_{1}, h_{1}\right)+\left\langle y^{*}, F_{1}(x, u)\right\rangle,
$$


where $\lambda_{0} \in \mathbf{R}, \lambda_{1} \in \mathbf{R}^{n}, y^{*} \in Y_{1}^{*}$. The function $\mathcal{L}$ can be represented in the form

$$
\begin{aligned}
\mathcal{E}\left(x, u, \lambda_{0}, \lambda_{1}, y^{*}\right)= & \lambda_{0}\left(\int_{0}^{T} f_{0}(t, x(t), u(t)) d t+\gamma_{2} S(u)\right)+\left(\lambda_{1}, x(0)-x_{0}\right) \\
& +\int_{0}^{T}(\psi(t), \dot{x}(t)-f(t, x(t), u(t)) d t,
\end{aligned}
$$

where $\psi(\cdot) \in L_{\infty}^{n}([0, T])$.

Applying the extremum principle, we obtain that a necessary condition for the point $\left(x_{*}, u_{*}\right)$ to be a solution to Problem IV is the existence of Lagrange multipliers $\lambda_{0} \geqslant 0, \lambda_{1}, y^{*}$, not vanishing simultaneously, for which

$$
\begin{gathered}
\mathcal{E}_{x}\left(x_{*}, u_{*}, \lambda_{0}, \lambda_{1}, y^{*}\right)=0, \\
\mathcal{L}\left(x_{*}, u_{*}, \lambda_{0}, \lambda_{1}, y^{*}\right)=\min _{u \in U_{z}} \mathcal{L}\left(x_{*}, u, \lambda_{0}, \lambda_{1}, y^{*}\right) .
\end{gathered}
$$

Hence

$$
\begin{aligned}
& \lambda_{0} \int_{0}^{T}\left(f_{0_{x}}\left(t, x_{*}(t), u_{*}(t)\right), \bar{x}(t)\right) d t+\left(\lambda_{1}, \bar{x}(0)\right) \\
& \quad+\int_{0}^{T}\left(\psi(t), \dot{\bar{x}}(t)-f_{x}\left(t, x_{*}(t), u_{*}(t)\right) \bar{x}(t)\right) d t=0
\end{aligned}
$$

for any $\bar{x}(\cdot) \in W_{1,1}^{n}([0, T])$, and

$$
\begin{aligned}
\int_{0}^{T}\left[\lambda_{0} f_{0}\left(t, x_{*}(t), u_{*}(t)\right)-\right. & \left.\left(\psi(t), f\left(t, x_{*}(t), u_{*}(t)\right)\right)\right] d t+\lambda_{0} \gamma_{2} S\left(u_{*}\right) \\
=\min _{u \in U_{z}}\left\{\int _ { 0 } ^ { T } \left[\lambda_{0} f_{0}\left(t, x_{*}(t), u(t)\right)\right.\right. & \\
& \left.\left.-\left(\psi(t), f\left(t, x_{*}(t), u(t)\right)\right)\right] d t+\lambda_{0} \gamma_{2} S(u)\right\} .
\end{aligned}
$$

Making use of (7), we may write condition (9) in the form

$$
\int_{0}^{T}(\psi(t), \dot{\bar{x}}(t)) d t-\int_{0}^{T}\left(H_{x}\left(\lambda_{0}, \psi, x_{*}, u_{*}, t\right), \bar{x}(t)\right) d t+\left(\lambda_{1}, \bar{x}(0)\right)=0
$$

Integrating the second addendum of (11) by parts, we get

$$
\int_{0}^{T}\left(\psi(t)-\int_{t}^{T} H_{x} d t, \dot{\bar{x}}(t)\right) d t+\left(-\int_{0}^{T} H_{x} d t+\lambda_{1}, \bar{x}(0)\right)=0
$$

Since the last equality holds for an arbitrary absolutely continuous function $\bar{x}(\cdot)$ therefore

$$
\psi(t)-\int_{t}^{T} H_{x} d t=\text { const, } \quad t \in[0, T]
$$


Hence it appears that $\psi(t)$ is absolutely continuous on $[0, T]$ and satisfies the differential equation

$$
\frac{d \psi}{d t}=-H_{x}\left(\lambda_{0}, \psi, x_{*}, u_{*}, t\right)=-f_{x}\left(t, x_{*}, u_{*}\right) \psi(t)+\lambda_{0} f_{0_{x}}\left(t, x_{*}, u_{*}\right) .
$$

Let us now come back to equality (12). It holds for any $\bar{x}(\cdot) \in W_{1,1}^{n}([0, T])$, in particular, for such that $\bar{x}(0)=0$. Then (12) has the form

$$
\int_{0}^{T}\left(\psi(t)-\int_{t}^{T} H_{x} d t, \dot{\bar{x}}(t)\right) d t=0 .
$$

Integrating by parts, we obtain

$$
\begin{aligned}
0 & =\int_{0}^{T}\left(\psi(t)-\int_{t}^{T} H_{x} d t, \dot{\bar{x}}(t)\right) d t \\
& =\left.\left(\psi(t)-\int_{t}^{T} H_{x} d t, \bar{x}(t)\right)\right|_{0} ^{T}-\int_{0}^{T}\left(\dot{\psi}(t)+H_{x}, \bar{x}(t)\right) d t \\
& =(\psi(T), \bar{x}(T)) .
\end{aligned}
$$

Since $\bar{x}(T)$ may assume any values, therefore $\psi(T)=0$. Consequently, the function $\psi$ satisfies the equation $\dot{\psi}=-H_{x}$ with the condition $\psi(T)=0$.

Condition (10) has the form

$$
\begin{aligned}
\int_{0}^{T}-H\left(\lambda_{0}, \psi, x_{*}, u_{*}, t\right) d t+\lambda_{0} \gamma_{2} S\left(u_{*}\right) \\
=\min _{u \in U_{z}}\left\{\int_{0}^{T}-H\left(\lambda_{0}, \psi, x_{*}, u, t\right) d t+\lambda_{0} \gamma_{2} S(u)\right\} .
\end{aligned}
$$

It follows from condition (13), assumption $\left(2^{\circ}\right)$ and the definition of the function $S$ that the function $u_{*}$ takes the values:

$$
u_{*}(t)= \begin{cases}\alpha^{1} & \text { when } H_{u}\left(\lambda_{0}, \psi(t), x_{*}(t), u_{*}(t), t\right)>0, \\ \beta^{1} & \text { when } H_{u}\left(\lambda_{0}, \psi(t), x_{*}(t), u_{*}(t), t\right)<0\end{cases}
$$

Since in Problem IV under consideration $u \in U_{z}$, the optimal control $u_{*}$ is a piecewise constant function.

Let us now estimate the number of points of discontinuity of the optimal control $u_{*}(\cdot)$.

Let $u_{0}(\cdot)$ be some admissible control. Thus the inequality

$$
I\left(x_{*}, u_{*}\right) \leqslant I\left(x_{0}, u_{0}\right)
$$


holds, where $x_{0}(\cdot)$ is a trajectory of system (3)-(4), corresponding to the control $u_{0}(\cdot)$. Hence

$$
\begin{aligned}
\int_{0}^{T} f_{0}\left(t, x_{*}(t),\right. & \left.u_{*}(t)\right) d t+\gamma_{1} N\left(u_{*}\right)+\gamma_{2} S\left(u_{*}\right) \\
& \leqslant \int_{0}^{T} f_{0}\left(t, x_{0}(t), u_{0}(t)\right) d t+\gamma_{1} N\left(u_{0}\right)+\gamma_{2} S\left(u_{0}\right) .
\end{aligned}
$$

Since $u_{*}(\cdot)$ is piecewise constant, therefore $S\left(u_{*}\right)=0$.

It follows from assumption $\left(1^{\circ}\right)$ that

$$
\alpha_{0}=\int_{0}^{T} \alpha(t) d t \leqslant \int_{0}^{T} f_{0}(t, x(t), u(t)) d t
$$

for an arbitrary admissible process $(x, u)$. Hence from (14) we obtain

$$
\gamma_{1} N\left(u_{*}\right)+\alpha_{0} \leqslant \int_{0}^{T} f_{0}\left(t, x_{0}(t), u_{0}(t)\right) d t+\gamma_{1} N\left(u_{0}\right)+\gamma_{2} S\left(u_{0}\right) .
$$

By $c$ let us denote a number equalling

$$
c=\int_{0}^{T} f_{0}\left(t, x_{0}(t), u_{0}(t)\right) d t+\gamma_{2} S\left(u_{0}\right) .
$$

We then obtain the following estimate of the number of points of discontinuity of $u_{*}(\cdot)$

$$
N\left(u_{*}\right) \leqslant N\left(u_{0}\right)+\frac{c-\alpha_{0}}{\gamma_{1}} .
$$

We have thus obtained the complete proof of Theorem 1 .

Let us now consider the case of a vector control. It is easy to prove the following.

Lemma 1. If $u_{*}(\cdot)=\left(u_{*}^{1}(\cdot), u_{*}^{2}(\cdot), \ldots, u_{*}^{r}(\cdot)\right)$ is an optimal control in Problem I, then each of the functions $u_{*}^{i}, i=1, \ldots, r$, is an optimal control in a scalar problem of the form

$$
\begin{aligned}
I_{1}\left(u^{i}\right) & =I\left(x, u_{*}^{1}, u_{*}^{2}, \ldots, u_{*}^{i-1}, u^{i}, u_{*}^{i+1}, \ldots, u_{*}^{r}\right) \rightarrow \inf , \\
\dot{x}(t) & =f\left(t, x(t), u_{*}^{1}(t), u_{*}^{2}(t), \ldots, u^{i}(t), \ldots, u_{*}^{r}(t)\right),
\end{aligned}
$$

where $u^{i}(\cdot) \in U^{i}$, and $U^{i}$ is a set defined as follows:

$$
U^{i}=\left\{u^{i}(\cdot) \text { piecewise continuous on }[0, T], u^{i}(t) \in\left[\alpha^{i}, \beta^{i}\right], S\left(u^{i}\right)<\infty\right\} \text {. }
$$

Repeating, for each $i=1,2, \ldots, r$, the proof of Theorem 1, we obtain the following; 
THEOREM 2. Let assumptions $\left(1^{\circ}\right)-\left(5^{\circ}\right)$ be satisfied. If, for each $i=1,2, \ldots, r$, $H_{u^{i}} \neq 0$ almost everywhere on $[0, T]$, then the optimal control for problem $I$, $u_{*}(\cdot)=\left(u_{*}^{1}(\cdot), u_{*}^{2}(\cdot), \ldots, u_{*}^{r}(\cdot)\right)$ is a piecewise constant function whose components $u_{*}^{i}(\cdot)$ assume the values $\alpha^{i}$ or $\beta^{i}$ only, and the number of points of discontinuity is estimated by formula $(8)$.

We shall now consider the case of a linear problem. Namely, assume that the functions $f$ and $f_{0}$ are of the form

$$
f=A x+B u, \quad f_{0}=a x+b u,
$$

where $A$ is a matrix of dimension $n \times n$ with constant coefficients, $B$ is a constant matrix of dimension $n \times r, a$ and $b$ are, respectively, $n$ - and $r$-dimensional vectors. It is easy to notice that, in this case, all earlier assumptions $\left(1^{\circ}\right)-\left(5^{\circ}\right)$ are satisfied.

For a linear problem, we shall now give a sufficient condition for $H_{u^{i}} \neq 0$, $i=1, \ldots, r$, to be satisfied.

THEOREM 3. If a system

$$
\tilde{x}(t)=\tilde{A} \tilde{x}(t)+\tilde{B} u(t),
$$

where $\tilde{x}=\left(x^{0}, x^{1}, \ldots, x^{n}\right), \dot{x}^{0}(t)=a x(t)+b u(t), \tilde{A}$ is a matrix of degree $(n+1)$ $\times(n+1)$ of the form

$$
\tilde{A}=\left[\begin{array}{cc}
0 & a^{1}, a^{2}, \ldots, a^{n} \\
0 & \\
\vdots & A \\
0 &
\end{array}\right],
$$

$\tilde{B}$ is a matrix of degree $(n+1) \times r$ of the form

$$
\tilde{B}=\left[\begin{array}{c}
b^{1} \ldots b^{r} \\
B
\end{array}\right]
$$

is regularly controllable ( $f f .[1])$, then, for each $i=1,2, \ldots, r$, the condition $H_{u^{i}} \neq 0$ is satisfied.

Proof. We have

$$
H=(\psi, A x+B u)+\lambda_{0}(a x+b u),
$$

where $\lambda_{0} \leqslant 0, \psi(t)$ is an absolutely continuous function and $\lambda_{0}$ and $\psi$ do not vanish simultaneously.

Let $\tilde{\psi}=\left(\lambda_{0}, \psi^{1}, \ldots, \psi^{n}\right)$. Then $H$ has the form

$$
H=(\tilde{\psi}, \tilde{A} \tilde{x}+\tilde{B} u)=(\tilde{\psi}, \tilde{A} \tilde{x})+(\tilde{\psi}, \tilde{B} u) .
$$


The function $\tilde{\psi}$ is a solution to the equation

$$
\dot{\psi}(t)=-H_{x}=-\tilde{A}^{*} \tilde{\psi}(t)
$$

Since $A$ is a constant matrix, therefore $\tilde{\psi}$ is an analytic function of the variable $t$. The derivative of the function $H$ with respect to $u$ has the form

$$
H_{u}=\tilde{B}^{*} \tilde{\psi}(t)
$$

Let us now suppose that there exists $j \in\{1,2, \ldots, r\}$ such that $H_{u^{j}}=0$ on set $Q \subset[0, T]$ of positive measure, i.e.

$$
\left(\tilde{b_{j}}, \tilde{\psi}(t)\right)=0 \text { for } t \in Q,
$$

where $\tilde{b}_{j}$ is the $j$ th column of the matrix $\tilde{B}$. The function $\left(\tilde{b_{j}}, \tilde{\psi}(t)\right)$ is analytic and vanishes on the set of positive measure, so

$$
\left(\tilde{b_{j}}, \tilde{\psi}(t)\right)=0 \text { for each } t \in[0, T] .
$$

Differentiating with respect to $t$, we have

$$
(\tilde{b}, \stackrel{\grave{\psi}}{\psi}(t))=0 .
$$

Taking account of (16), we get

$$
\left(\tilde{b_{j}},-\tilde{A}^{*} \tilde{\psi}(t)\right)=0
$$

that is,

$$
\left(\tilde{A} \tilde{b}_{j}, \tilde{\psi}(t)\right)=0 .
$$

Further, proceeding analogously as in the proof of Theorem 6.5 (cf. [1]), we come to a conclusion that system (15) is not regularly controllable. This contradicts the assumption of the theorem. Consequently, $H_{u^{i}} \neq 0$ for $i=1,2, \ldots, r$.

To close with, we shall give an example illustrating the theorem we have proved.

EXAMPLE. In the space $\mathbf{R}^{2}$ let us consider a control system of the form

$$
\begin{aligned}
\dot{x}^{1} & =\frac{1}{2} x^{2}+\frac{1}{3} u^{1}, \\
\dot{x}^{2} & =\frac{1}{3} x^{1}+\frac{1}{2} x^{2}+\frac{1}{4} u^{2}, \\
x^{1}(0) & =x^{2}(0)=0, \quad\left|u^{1}\right| \leqslant 1,\left|u^{2}\right| \leqslant 1,
\end{aligned}
$$

with a cost functional defined by the formula

$$
I(x, u)=\int_{0}^{1}\left(-x^{1}-x^{2}+\frac{1}{3} u^{1}\right) d t+N(u)+S(u),
$$

where $x=\left(x^{1}, x^{2}\right), u=\left(u^{1}, u^{2}\right)$. 
It is easy to see that in this example the system $\dot{x}=\tilde{A} \tilde{x}+\tilde{B} u$ is of the form (cf. Theorem 3)

$$
\left[\begin{array}{c}
\dot{x}^{0} \\
\dot{x}^{1} \\
\dot{x}^{2}
\end{array}\right]=\left[\begin{array}{ccc}
0 & -1 & -1 \\
0 & 0 & \frac{1}{2} \\
0 & \frac{1}{3} & \frac{1}{2}
\end{array}\right]\left[\begin{array}{l}
x^{0} \\
x^{1} \\
x^{2}
\end{array}\right]+\left[\begin{array}{cc}
\frac{1}{3} & 0 \\
\frac{1}{3} & 0 \\
0 & \frac{1}{4}
\end{array}\right]\left[\begin{array}{l}
u^{1} \\
u^{2}
\end{array}\right] .
$$

By putting, successively, $u^{1}=0$ and $u^{2}=0$, it can easily be checked that system (19) is regularly controllable. From Theorem 3 it follows that in this example the assumptions of Theorem 2 are satisfied. Making use of the Cauchy formula, we obtain

$$
\begin{aligned}
|x(t)| & =\left|\int_{0}^{t} e^{A(\tau-t)} B u(\tau) d \tau\right| \\
& \leqslant e^{\|A\| \|}\|B\|\|u\| \leqslant e \cdot \frac{1}{5} \cdot \sqrt{2} \leqslant 1 \quad \text { for } t \in[0,1],
\end{aligned}
$$

where

$$
A=\left[\begin{array}{cc}
0 & \frac{1}{2} \\
\frac{1}{3} & \frac{1}{2}
\end{array}\right], \quad B=\left[\begin{array}{cc}
\frac{1}{3} & 0 \\
0 & \frac{1}{4}
\end{array}\right] .
$$

Hence

$$
f_{0}(t, x, u)=-x^{1}-x^{2}+\frac{1}{3} u^{1} \geqslant-2 \frac{1}{3} .
$$

And consequently, for $\alpha_{0}$ in formula (8) we may take $\alpha_{0}=-2 \frac{1}{3}$. Put $u_{0} \equiv 0$. It follows from (17) and (19) that

$$
c=\int_{0}^{1} f_{0}\left(t, x_{0}(t), u_{0}(t)\right) d t+\gamma_{2} S\left(u_{0}\right)=0 .
$$

Using Theorem 2, we infer that each component of the optimal control $u_{*}=$ $\left(u_{*}^{1}, u_{*}^{2}\right)$ is piecewise constant, assumes the values \pm 1 and possesses at most two jumps.

\section{References}

[1] M. Athans and P. L. Falb, Optimal control (McGraw-Hill, New York, 1966).

[2] A. D. Ioffe and V. M. Tikhomirov, Theory of extremal problems (North-Holland, New York, 1979).

[3] E. S. Noussair, "On the existence of piecewise continuous optimal controls", J. Austral. Math. Soc. Ser. B. 20 (1977), 31-37. 